\title{
Deciphering DNA methylation signatures of pancreatic cancer and pancreatitis
}

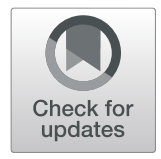

Francesco Natale ${ }^{1 *}$, Maria Vivo $^{1}$, Geppino Falco ${ }^{1,2}$ and Tiziana Angrisano ${ }^{1 *}$ (D)

\begin{abstract}
Background: Chronic pancreatitis presents a high risk of inflammation-related progression to pancreatic cancer. Pancreatic cancer is the fourth leading cause of cancer-related death worldwide. The high mortality rate is directly related to the difficulty in promptly diagnosing the disease, which often presents as overt and advanced. Hence, early diagnosis for pancreatic cancer becomes crucial, propelling research into the molecular and epigenetic landscape of the disease.

Main body: Recent studies have shown that cell-free DNA methylation profiles from inflammatory diseases or cancer can vary, thus opening a new venue for the development of biomarkers for early diagnosis. In particular, cell-free DNA methylation could be employed in the identification of pre-neoplastic signatures in individuals with suspected pancreatic conditions, representing a specific and non-invasive method of early diagnosis of pancreatic cancer. In this review, we describe the molecular determinants of pancreatic cancer and how these are related to chronic pancreatitis. We will then present an overview of differential methylated genes in the two conditions, highlighting their diagnostic or prognostic potential.

Conclusion: Exploiting the relation between abnormally methylated cell-free DNA and pre-neoplastic lesions or chronic pancreatitis may become a game-changing approach for the development of tools for the early diagnosis of pancreatic cancer.
\end{abstract}

Keywords: Chronic pancreatitis, Pancreatic cancer, Cell-free DNA, DNA methylation, Diagnostic methods, Preneoplastic lesions

\section{Background}

Chronic pancreatitis (CP) represents a spectrum of persisting fibro-inflammatory disorders of the exocrine pancreas that alter the organ's typical structure and functions, and significantly reduce patients' quality of life [1]. Genetic predisposition [2, 3], neoplasms, intraductal obstruction, or autoimmune pancreatitis (AIP) can all cause CP [4]. Alcohol history is considered a significant risk factor, and tobacco smoking may act synergistically with alcohol [5]. CP affects $\sim 50$ per 100,000 individuals worldwide, with an incidence that is expected to increase over time [5]. The disease often presents with upper abdominal pain, nausea or vomiting, and steatorrhea. The diagnosis is based on tests on pancreatic function or structure, such as elevated amylase or lipase serum levels, secretin stimulation, or computed tomography.

\footnotetext{
* Correspondence: francesco.natale.bio@gmail.com; tangrisa@unina.it ${ }^{1}$ Department of Biology, University of Naples Federico II, 80126 Naples, Italy Full list of author information is available at the end of the article
}

The persistent inflammatory state in $\mathrm{CP}$ promotes accelerated tissue repair, which may result in neoplastic (trans)formation. For this reason, CP represents one of the highest risk factors for the development of pancreatic tumors (PTs) [6, 7]. PT is an aggressive disease usually asymptomatic at an early stage displaying symptoms resembling $\mathrm{CP}$ once it is overt [8]. This feature hinders early diagnosis, contributing to high observed mortality (5-year survival: 6\%) $[9,10]$. The global incidence rate of PT in 2012 was about six per 100,000 individuals [9] and, coherently with $\mathrm{CP}$, it is expected to increase over time.

In the absence of an underlying PT, the lag period between $\mathrm{CP}$ diagnosis and tumor is usually one or two decades $[11,12]$. This long latency period might offer a strategic opportunity for early diagnosis and curative treatment once biomarkers with robust predictive power are discovered.

(c) The Author(s). 2019 Open Access This article is distributed under the terms of the Creative Commons Attribution 4.0 International License (http://creativecommons.org/licenses/by/4.0/), which permits unrestricted use, distribution, and 
The best-known biomarker for PT is the serum proteincarbohydrate antigen 19-9 (CA19-9, or sialylated Lewis antigen) [13]. When released by a PT, CA19-9 levels can help to monitor the treatment or the relapse of the disease [14]. Unfortunately, elevated CA19-9 levels are also present in CP or other cancers [15]. Besides, about 10\% of the Caucasian population lacks CA19-9 on their red blood cells [15]. Due to these limitations, the American Society of Clinical Oncology discouraged the use of CA19-9 as a biomarker for PT diagnosis [14]. The lack of robust noninvasive diagnostic screening methods has propelled the research of potential biomarkers in patients' biological fluids. Sequence analysis of cell-free DNA (cfDNA) from the bloodstream of patients affected by PT uncovered mutations in the KRAS already in the early 90s [16], and improvement of these methods was attained in the past years $[2,17]$. A more recent and emerging field investigates the methylation levels of circulating cfDNA. This approach is actively used for the discovery of biomarkers of several diseases [18-20]. Its popularity stems from the increasing evidence that cfDNA carries methylation marks that enable the identification of tissue-specific cell death [21] and are more broadly informative, sensitive, and specific than individual DNA mutations [22-24]. Further, sample collection is minimally invasive and allows adequate followup under negligible stress conditions for the patient.

In this review, we provide an overview of the molecular determinants of PT and the genes showing differential DNA methylation in $\mathrm{CP}$, pre-neoplastic lesions of the pancreas, and PT. In particular, we will compare studies conducted either on primary tissue biopsies or from biological fluids. We aim to decipher disease-specific methylation patterns in pancreatic diseases to serve as a novel diagnostic or prognostic tool for PT. The identification of a "pancreatic disease signature" to distinguish between inflammation and cancer could hopefully enhance non-invasive tools for the early diagnosis of PTs.

\section{The molecular landscape of pancreatic cancer and pancreatitis}

The pancreatic adenocarcinoma develops almost exclusively from the exocrine pancreatic ductal epithelium cells accounting for $85 \%$ of all PTs. Other PTs include the acinar cell carcinoma originating from the exocrine acini of the pancreas, the pancreatic neuroendocrine tumors (PNETs) arising from neuroendocrine cells, and other minorities. For its abundance, we will hereafter use the term PT to refer to pancreatic ductal adenocarcinoma, unless otherwise specified.

Molecularly, a variety of genetic and epigenetic events underlie the development of PT (Fig. 1).

Pre-neoplastic non-invasive lesions are thought to be the first stage of PT etiopathogenesis. Low-grade pancreatic intraepithelial neoplasia (PIN) is the most frequent microscopic lesion of the pancreas, harboring mutations in the KRAS in about $90 \%$ of the cases $[8,32]$. As the grade of the lesion progresses, additional mutations in

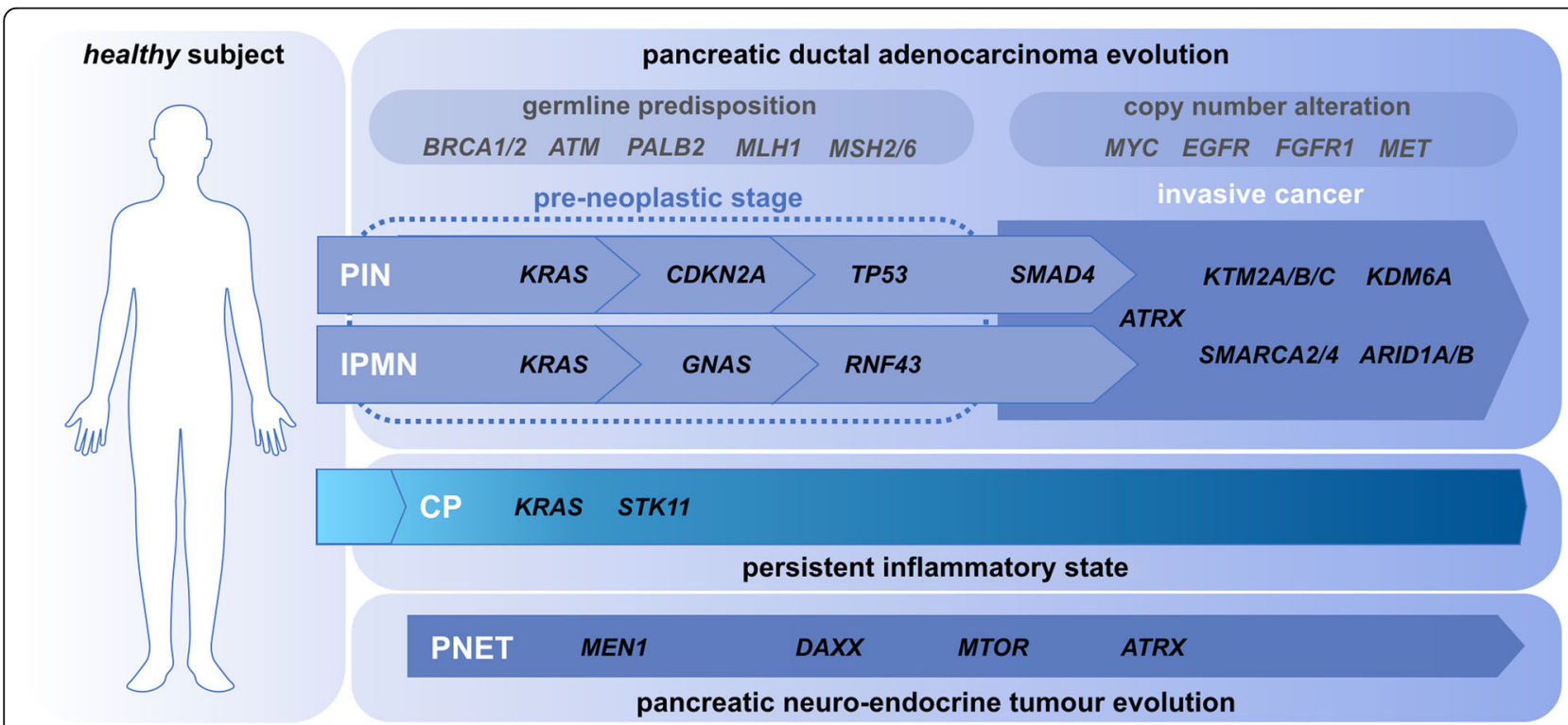

Fig. 1 Genetic landscape of pancreatic cancer. Genes mutated in pancreatic ductal adenocarcinoma (PT, top) and pancreatic neuroendocrine tumor (PNET, bottom). For the former, genetic predisposition or copy number alterations are also reported (reviewed in [25]). At the preneoplastic stage (dotted line), pancreatic intraepithelial neoplasias (PIN) and intraductal papillary mucinous neoplasms (IPMN) coexist. Overall, aberrant homeostasis of genes regulating cell differentiation, proliferation and apoptosis promote the transition from pre-neoplastic to advanced stages of the disease [26-31]. Chronic pancreatitis (CP) may also be present, contributing to the progression of an underlying tumor 
cell cycle-regulating CDKN2A and TP53 are found [8]. Intraductal papillary mucinous neoplasms (IPMNs) are macroscopic lesions and have a $25 \%$ risk of developing into invasive PT. Other than KRAS, they often present mutations in genes involved in the Wnt signalling pathway (e.g., GNAS and RNF43) [8]. Other types of lesions include mucinous cystic neoplasms and intraductal tubulopapillary neoplasms [32, 33].

Genes mutated in PNETs are generally different from those found in exocrine PTs [34]. For example, KRAS mutation is absent in most PNETs, while frequent mutations in these tumors occur in DAXX (encoding the transcriptional corepressor death domain-associated protein 6), MTOR (encoding the mammalian target of rapamycin kinase), and MEN1 genes [35].

At the invasive stage, PTs present mutations in SMAD4 (about 55\% of the cases) and genes of the SWI/ SNF chromatin remodeling family ( $15 \%$ of the cases) $[6,8]$. A member of this family, ATRX, is frequently mutated in PNETs [35], and these events have been shown to cause changes in DNA methylation patterns [36, 37]. The latter, together with aberrant DNA hydroxymethylation, are established processes contributing to cancer development [38-41].

Inflammatory stimuli may lead to aberrant DNA methylation homeostasis and, coherently, to gene expression changes [39, 42-44]. Further, the progressive increase of DNA methylation levels has been described in chronic inflammatory diseases developing into cancer. The interactions between inflammation and epigenetics in tumor initiation, promotion, and immune evasion can be leveraged in cancer prevention and treatment [40]. Thus, it is not surprising that the relative risk for $\mathrm{CP}$ patients developing PT is generally $>10[11,45]$. In this scenario, chronic inflammation may inactivate the oncogene-induced senescence barrier that is typical for preneoplastic PIN lesions and, thus, it promotes the neoplastic progression of PT [46]. Genetically, $4-28 \%$ of CP cases show KRAS mutation [2, 3].

Conversely, no KRAS mutation at known tumor-promoting sites has so far been observed in AIP [47]. Finally, the mutation of the STK11, a known risk factor for PTs, has recently been described in CP [17]. In the past two decades, a significant number of studies conducted in pancreatic cell lines, xenografts, or primary tissue specimens aimed at identifying aberrant DNA hyper- or hypomethylation targets in PTs. Almost all DNA methylation analyses rely on PCR-based methods, on bisulfitetreated-or not-specimens [48]. For their high throughput, microarray or-more recently-next-generation sequencing (NGS) methods enabled the discovery of many target genes. The fast and cost-containing methylationspecific PCR (MSP) is generally the method of choice for target validation. This approach led to the identification of many candidate genes associated with epigenetic changes taking place during the carcinogenesis of PT. In this section, we will discuss those genes whose DNA methylation levels hold potential for the differential diagnosis of $\mathrm{CP}$, pre-neoplastic conditions of the pancreas, and invasive PTs. Besides, the genes, as well as the method employed for their discovery and validation, will be described.

\section{Differentially DNA-methylated genes in pancreatic tumor and pancreatitis}

The first studies comparing cancerous and healthy pancreatic tissues analyzed the methylated status of known tumor-suppressors and cancer-associated genes. Also, specific CpG islands spread within the genome were found preferentially methylated in tumors (MINT loci). In particular, the DNA methylation status of $\mathrm{CpG}$ islands embedded in several gene promoters (CACNA1G, CDH1, CDKN2A, DAPK1, MGMT, MINT1-2-31-32, MLH1, $R A R B, T H B S 1$, and TIMP3) was analyzed in PT xenografts $[49,50]$. All cancerous specimens showed aberrant DNA methylation of at least one locus except for MGMT, which was non-methylated in either neoplastic or normal samples. Simultaneous methylation of at least four loci was a feature of $\sim 14 \%$ of PT xenografts. Overall, the most frequently methylated loci were MINT32, MINT1, and MINT28. Moreover, seven CpG islands (CGIs), of which three associated to the known CCNG1, PENK, ZBP genes, were found differentially methylated in pancreatic-derived cell lines compared with the healthy pancreas and validated in PTs [51, 52]. Among these PENK, CGI was found methylated in $91 \%$ of cases. Of note, five specimens of $\mathrm{CP}$ were also analyzed, showing PENK CGI methylation in two cases [52].

PENK, as well as $C D K N 2 A$, DNA methylation status was further investigated in PINs of different grade: intraductal PTs, extra-ductal PTs (including one PNET), and CP specimens [53, 54]. PENK methylation was present in $93 \%$ of invasive PTs, of which $27 \%$ presented CDKN2A methylated. In contrast, non-neoplastic specimens resected from matched healthy tissues did not harbor methylation of either gene. Noteworthy, the prevalence of PENK methylation increased significantly with increasing lesion grade (from 8 to $46 \%$, in grade 1A or grade 3 lesions, respectively). Only one of the extra-ductal PTs presented CDKN2A methylation, while $\mathrm{CP}$ specimens had neither gene methylated. Interestingly, as regarding $C D K N 2 A$, available data suggest that hypermethylation associated with the loss of CDKN2A expression might occur only in CP in which low-grade PINs were observed [45]. In line with its broad role as a tumor suppressor, the downregulation of CDKN2A expression was found in this study independently of methylation status. 
These data support the disease stage-specific DNA methylation model and qualify these genes as potential biomarkers of early pancreatic carcinogenesis.

Comparing invasive PTs to IPMNs, 6\% of IPMNs and $22 \%$ of invasive PTs presented SOCS1 methylation. In contrast, none of the pancreatic normal ductal epithelia and the PINs that were analyzed presented SOCS1 methylation [55]. In a preliminary study, the analysis of IPMNs with different grades of invasiveness reported SOCS1 methylation in 6\% of invasive IPMNs [56], providing evidence that, therefore, this gene might be an early indicator of invasiveness of the disease. About half of the tested specimens scored positive for CDKN2A (p16INK4a specific region) and TP73 methylation, independently of their grade of invasiveness. Conversely, CDKN2B, ESR1, and TIMP3 were methylated in none of the examined specimens. More frequent methylation of APC $(50 \%$ vs. $10 \%$ ), $C D H 1$ ( $38 \%$ vs. $10 \%$ ), $M G M T$ ( $45 \%$ vs. $20 \%$ ), and MLH1 (38\% vs. 10\%) was observed in invasive IPMNs, compared to non-invasive samples. Unfortunately, the weakness of these findings results from the limiting size of the non-invasive IPMN subpopulations [45].

A progressive increase of the DNA methylation of at least one gene and the average number of methylated genes was observed from PIN to PT in 58 patients who had undergone resection surgery for invasive PTs.

The impact of the inflammatory environment on DNA methylation was assessed. The data showed that DNA methylation also increases with inflammation of pancreatic tissue. BRCA1, APC, CDKN2A, and TIMP3 were methylated in $60 \%, 59 \%, 39 \%$, and $31 \%$ of the PT cases, respectively [57]. Finally, the DNA methylation levels of six genes frequently hypermethylated in PT (CCND2, CDKN2A, FOXE1, NPTX2, PENK, TFPI2) were analyzed in AIP specimens [47], showing no significant hypermethylation in both AIP or healthy pancreas.

Evidence of DNA hypomethylation events in PTs is also reported [58-60]. A panel including 18 genes known to be over-expressed in PTs and 14 genes whose overexpression in PT was not documented was composed. MSP analysis revealed methylation of 19 of 32 genes in healthy pancreas. All genes that were known to be transcribed at high levels in PTs but not expressed in healthy pancreas (CLDN4, LCN2, MSLN, PSCA, S100A4, $T F F 2$, and YWHAS) were frequently hypomethylated in PT xenografts. In particular, five or six genes showed simultaneous DNA hypomethylation in $92 \%$ or $61 \%$ of the cases, respectively. Moreover, from 379 identified loci hypomethylated in PT with respect to healthy pancreas [61], the oncogenes FOS, JUNB, and $M Y B$; the genes NDN and SMARCA1 [62]; and the chromatin modifiers genes such as CTR9, EP400, HIRIP3, KDM6A, $K M T 5 A$, and PRMT1 were identified. These genes presented increased expression in PT specimens playing a role in core signalling pathways of PT [60].

In Additional file 1: Table S1, we reported a list of several studies that analysed the methylation status of different genes. Genes, commonly addressed in both primary tissues and liquid biopsies (see next section), were grouped and highlighted in gray.

\section{Genome-wide profiling of methylated genes in pancreatic tumor and pancreatitis}

The advent of genomic technologies improved the screening capability by several orders of magnitude, providing promising results. Using a high-throughput microarray approach, 11 genes (CDH3, CLDN5, FOXE1, LHX1, NPTX2, RPRM, SFRP1, ST14, TJP2, UCHL1, and WNT7A) markedly induced after treatment with 5-aza2-deoxycytidine (5Aza-dC), a DNA demethylation agent, were specifically identified in pancreatic tumor cell lines [63]. Analysis of the methylation status of these genes in PTs showed that four genes (CLDN5, NPTX2, SFRP1, $U C H L 1)$ were methylated in at least $93 \%$ of the screened specimens, while only two (CDH3 and ST14) presented methylation in less than $20 \%$ of samples.

A microarray-based method was used to interrogate 27,800 CGIs covering 21 Megabase-pairs of the human genome. Then, 1968 CGIs showed differential methylation in pancreatic cancer cell lines compared to a healthy pancreas. Validation in 57 PTs and 34 normal pancreases confirmed specific methylation of $M D F I$, MIR9-1, ZNF415, CNTNAP2, and ELOVL4 in cancer-derived samples [64].

Further application of this strategy resulted in the identification of $>1200$ known loci that presenting specific methylation in PT. Many of these loci belonged to Wnt signalling pathway or the homeobox and the cadherin superfamilies [61]. Analysis of 24 of these hypermethylated candidate genes (ADCY5, BMI1, BTBD6, CACNA1H, EFNA4, FOXF2-G1, FZD1-2-7, HIC1, ID4, IRF5, NEUROG3, PCDH17, PDE4B, SFRP1, SIM1, SMOC2, SOX3-15, WNT3-5A, ZBTB16) was validated in PT showing that for 20 genes DNA methylation was, on average, $50 \%$ more frequent in PTs. Only for EFNA4, BTBD6, and FZD2-7 DNA methylation levels were comparable between the two conditions [61]. A transcriptomics-based screening of pancreatic cell lines identified-from a pool of $>1400$ genes-eight potential biomarkers showing PT-specific DNA methylation signatures [65]. This gene-panel was then used to probe a large cohort of PT, CP, and healthy pancreas specimens. The most frequently methylated genes in PTs were $B N C 1$ and ADAMTS1. Quantitative MSP (qMSP) validation confirmed the results. Interestingly, $B N C 1$ methylation was detected in PINs, while ADAMTS1 methylation was exclusively found in invasive PTs. 
Importantly, these two genes showed increased DNA methylation in pre-neoplastic PINs, with little to no DNA methylation in CP or healthy pancreas [65], thus underlining their potential use for early PT diagnosis and prognosis. In a comprehensive program, composed of four case-control studies, PT-specific DNA-methylated markers were further identified through NGS [22]. Analysis of $>1,000,000 \mathrm{CpG}$ sites derived from matched PTs, benign pancreas, and healthy colon tissue specimens resulted in the identification of $>500$ DNA-methylated regions (DMRs). Interestingly, upon biological validation, six of 87 candidate genes (CD1D, CLEC11A, IKZF1, KCNK12, NDRG4, PRKCB) were further selected and tested as diagnostic biomarkers in cfDNA specimens (as described in next section) [22].

NGS was also utilized to identify differentially DMRs in healthy and neoplastic samples. Aberrantly methylated CGIs were more frequent in PT, compared to the healthy pancreas, and DNA hypermethylation events in PTs typically occurred in the vicinity of the transcription start site (TSS). Several individual DMRs (including C5orf38, DLX4, ELAVL2, EMX1, IRX1, NPR3, PITX2, SIM2, TBX5, TFAP2C, and VSTM2B) were further validated by target-specific methods (MSP, direct bisulfite sequencing or methylation-sensitive restriction endonuclease PCR) [58]. It is well-known that DNA methylation can occur in diverse genomic contexts such as promoters, CGIs and CGI shores, introns, exons, and miRNAs. Epigenetic deregulation of non-coding genes like microRNAs (miRs) is an established event in tumor development. miRNA are commonly involved in inflammatory processes playing a role in coordinating several features of the immune system, including immune cell differentiation, function, and recruitment.

Three MIR124 genes and MIR1OB showed a higher mean CpG methylation fraction in PTs compared with matched non-cancerous tissues. Hypermethylation induced the silencing of MIR124, which was associated with poor prognosis. The functional implications of such findings derive from MIR124 role in inhibiting cell proliferation, invasion, and metastasis. Among MIR124 direct targets, stem Rac1, a putative PT-promoting factor that activates JNK-dependent cell motility [66-70].

Also, MIR210 and MIR130B displayed PT-specific DNA-hypomethylation. Supporting evidence relates to high levels of MIR210 to PT progression, epithelial-tomesenchymal transition, and adverse prognosis in cancer patients [71, 72]. For MIR130B, its DNA methylation levels [73] and the relative functional implications are still controversial: while expression of $M I R 130 B$ is reported to inhibit cell proliferation and invasion in PTs by direct targeting of STAT3 [74], increased levels of this miR associates with the development of other neoplastic diseases [75].

\section{Pancreatic cancer and pancreatitis cfDNA methylation signatures in biological fluids}

To find a diagnostic or prognostic tool relevant for PT, several studies, rather than analyzing only one gene, combined many candidate genes into aggregate biomarkers. The methylation levels of the different genes in the aggregate were adopted to differentially diagnose pre-neoplastic conditions or PT from different biological fluids, such as pancreatic juice and blood, as indicated in Table 1.

While in PT solid biopsies, six methylated genes were identified to be predictive of advanced disease; in pancreatic juice, only three of them (CLDN5, NPTX2, and SFRP1) showed the potential to be employed as aggregate biomarkers [63]. Indeed, aberrant DNA methylation of at least one of the three was detectable in $75 \%$ of the pancreatic juice specimens from PT and in none of the benign samples. The absence of DNA methylation for all three genes was observed in $16 \%$ of PTs, resulting in a specificity of $75 \%$. Of note, four PNETs were also probed and displayed no DNA methylation. These results were encouraging, and pancreatic juice from larger cohorts was examined in other studies.

For example, Matsubayashi et al. investigated pancreatic juice endoscopically or surgically collected from individuals with suspected pancreatic diseases, including $\mathrm{CP}$ and benign pancreatic lesions [76]. Quantitative interrogation of 17 target genes revealed increased DNA methylation levels in all PT specimens $(n=56)$, compared to the normal pancreas $(n=11)$. Further, the number of methylated genes in the high-risk PT group $(n=44)$ was more abundant than in healthy controls but similar to patients with chronic pancreatitis $(n=11)$. Combination of the five most discriminating assays (CCND2, FOXE1, NPTX2, PENK, and TFPI2) scoring DNA methylation in more than one gene was highly predictive of PT: nine of 11 patients with PT but none of the 64 individuals without detectable pancreatic neoplasia presented DNA methylation signal (82\% sensitivity and $100 \%$ specificity for PT). Interestingly, these genes were previously identified in several studies conducted on pancreatic biopsies. Although none of the target genes was useful to distinguish $\mathrm{CP}$ from healthy pancreases, this aggregate biomarker successfully discriminated PTs from other pancreatic lesions.

In their clinical pilot testing, Kisiel et al. assessed the DNA methylation levels of six previously identified candidate biomarkers, together with mutant KRAS status, on secretin-stimulated pancreatic juice samples from $61 \mathrm{PT}$ patients, $22 \mathrm{CP}$ patients, and 19 with healthy pancreas [22]. In line with previous findings, mutant KRAS had a sensitivity of $56 \%$ and $39 \%$ (at $90 \%$ specificity) in discriminating PTs or CP from healthy pancreases, respectively. Overall, the other biomarkers 
Table 1 List of cfDNA methylation aggregate biomarkers with high sensitivity for chronic pancreatitis or pancreatic cancer diagnosis

\begin{tabular}{|c|c|c|c|c|c|c|}
\hline \multicolumn{2}{|c|}{ Aggregate biomarkers } & \multirow{3}{*}{$\begin{array}{l}\text { Comparison } \\
\text { CP vs. PT }\end{array}$} & \multirow{3}{*}{$\begin{array}{l}\text { Diagnostic } \\
\text { method } \\
\text { MSP }\end{array}$} & \multicolumn{2}{|c|}{ Test performance $(95 \% \mathrm{Cl})$} & \multirow{3}{*}{$\begin{array}{l}\text { Reference } \\
\text { Sato et al. 2003a [59] }\end{array}$} \\
\hline & & & & \multirow{2}{*}{$\begin{array}{l}\text { Combined sensitivity } \\
75 \%\end{array}$} & \multirow{2}{*}{$\begin{array}{l}\text { Combined specificity } \\
75 \%\end{array}$} & \\
\hline CLDN5 & NPTX2 & & & & & \\
\hline \multicolumn{7}{|l|}{ SFRP1 } \\
\hline CCND2 & FOXE1 & $N+C P$ vs. PT & qMSP & $82 \%$ & $100 \%$ & Matsubayashi et al. 2006 [76] \\
\hline NPTX2 & PENK & & & & & \\
\hline \multicolumn{7}{|l|}{ TFPI2 } \\
\hline CCND2 & PLAU & N vs. PT & MCAM & $76 \%$ & $59 \%$ & Melnikov et al. 2009 [77] \\
\hline SOCS1 & THBS1 & & & & & \\
\hline \multicolumn{7}{|l|}{ VHL } \\
\hline$B R C A 1$ & CCND2 & N vs. CP & MCAM & $82 \%$ & $78 \%$ & Ligget et al. 2010 [80] \\
\hline CDKN1C & MLH1 & & & & & \\
\hline$P G R$ (distal) & $P G R$ (proximal) & & & & & \\
\hline SYK & VHL & & & & & \\
\hline CDKN1C & CDKN1B & CP vs. PT & MCAM & $91 \%$ & $91 \%$ & Ligget et al. 2010 [80] \\
\hline CCND2 & DAPK1 & & & & & \\
\hline $\operatorname{ESR1}(A)$ & MGMT & & & & & \\
\hline MLH1 & MUC2 & & & & & \\
\hline MYOD1 & PGK1 & & & & & \\
\hline$P G R$ (proximal) & RARB & & & & & \\
\hline$R B 1$ & SYK & & & & & \\
\hline ADAMTS1 & $B N C 1$ & N vs. PT & $\mathrm{qPCR}$ & $81 \%(69 \%-93 \%)$ & $85 \%(71 \%-99 \%)$ & Yi et al. 2013 [65] \\
\hline$A P C$ & BMP3 & CP vs. PT & MSP & $76 \%$ & $83 \%$ & Henriksen et al. 2016 [82] \\
\hline$B N C 1$ & MESTV2 & & & & & \\
\hline RASSFIA & SFRP1 & & & & & \\
\hline SFRP2 & TFPI2 & & & & & \\
\hline Age $(>65)$ & & & & & & \\
\hline
\end{tabular}

CP chronic pancreatitis, PT pancreatic adenocarcinoma, $N$ normal. The gene names' was included in gene names' abbreviation section

performed better: the sensitivity (at $90 \%$ specificity) in discriminating PT from healthy pancreas was $79 \%$ for $C D 1 D, 67 \%$ for CLEC11A, $62 \%$ for IKZF1, 79\% for KCNK12, $72 \%$ for NDRG4, and $67 \%$ for PKRCB. The sensitivity (at $90 \%$ specificity) in discriminating PT from CP was generally lower: $53 \%$ for CLEC11A, $54 \%$ for IKZF1, $46 \%$ for KCNK12, and $67 \%$ for NDRG4. Of note, $C D 1 D$ displayed a better discriminating potential between PT and CP (84\%), while PKRCB methylation was worse $(38 \%)$ than mutant KRAS at distinguishing PTs from CPs.

The collection of pancreatic juice is a relatively complex procedure, and it might not be appropriate as a standard approach for early detection in asymptomatic individuals. For this, the analysis of cfDNA in plasma specimens received increasing inputs.

Melnikov et al. examined plasma specimens of PT patients with a panel of 56 frequently methylated genes [77]. By mean of a microarray-based approach [78], five promoters were selected (CCND2, PLAU, SOCS1,
THBS1, and $V H L)$ and combined in an aggregate biomarker. This test assesses DNA hypomethylation events, which makes comparison with techniques that detect cancer-related hypermethylation difficult. The assay distinguished healthy pancreas from PTs with $76 \%$ sensitivity and $59 \%$ specificity. Previous findings had reported CCND2, SOCS1, and THBS1 hypermethylation as informative for cancer detection in pancreatic juice or tissues $[52,55,79]$.

In a follow-up study by Liggett et al., 17 gene promoters were examined to identify differential cfDNA methylation in PT and $\mathrm{CP}$ patients [80]. Eight genes (BRCA1, CCND2, CDKN1C, MLH1, proximal and distal $P G R$ promoter regions, $S Y K$, and $V H L$ ) were useful to distinguish healthy pancreas from $\mathrm{CP}$, with a sensitivity and a specificity of $82 \%$ and $78 \%$, respectively. For all genes in this panel, promoter DNA methylation was more frequent in individuals with $\mathrm{CP}$. Fourteen genes (CCND2, CDKN1C, CDKN2B, DAPK1, promoter A of ESR1, MGMT, MLH1, MUC2, MYOD1, PGK1, the 
proximal region of the $P G R$ promoter, $R A R B, R B 1$, and $S Y K)$ were able to distinguish CP from PTs with a sensitivity and a specificity of $91 \%$. It is worth noting that all genes that were hypermethylated in $\mathrm{CP}$ displayed hypomethylation in PT. As CP often precedes PT, dynamic DNA methylation patterns for a given set of genes might underlie the progression of the disease. Validation in larger independent cohorts would be critical to confirm these findings.

Park et al. investigated the DNA methylation levels of six genes (CDKN2A, NPTX2, PENK, SFRP1, RASSF1A, and $U C H L 1)$ in the plasma of individuals with PT and CP [81]. The investigated genes had previously been reported to present high DNA methylation in $81 \%$ of PTs (13 of 16 cases), $61 \%$ of CP (eight of 13 cases), with more than one gene affected in either condition. In contrast, less than $4 \%$ of healthy pancreases (one of 29) presented DNA methylation. In this study, high interindividual variability was observed, and significant differences between PT and CP could not be confirmed, except for $C D K N 2 A$, specifically methylated in PT but not in CP.

Scarcely abundant cfDNA is often a limiting factor for many studies. To overcome such issue and improve the sensitivity of DNA methylation detection, $\mathrm{Yi}$ et al. employed a single-tube high-yield collection method, termed methylation on beads (MOB) [65]. Based on their previous screening, the authors determined the sensitivity assuming that all patients with PT (stages IIV; $n=42$ ) would harbor $B N C 1$ and ADAMTS1 gene methylation while healthy subjects $(n=26)$ would not [65]. Further, 33 of $42 \mathrm{PT}$ patients showed BNC1 methylation, while 20 of 42 showed ADAMTS1 methylation, with a sensitivity of $79 \%$ and $48 \%$ for $B N C 1$ and ADAMTS1, respectively. Specificity was $89 \%$ for $B N C 1$ and $92 \%$ for ADAMTS1. Combining both genes slightly improved the overall sensitivity $(81 \%)$ but worsened the overall specificity (85\%).

A recent prospective study from Henriksen et al. employed a panel of 28 genes to assess the DNA methylation state in patients with PT $(n=95), \mathrm{CP}(n=124)$, and acute pancreatitis $(n=59)$ from plasma specimens [82]. Compared with $\mathrm{CP}$, patients with $\mathrm{PT}$ presented more methylated genes, on average (8.4 vs. 4.7). The authors developed a diagnostic prediction model that optimized the combination of biomarkers to achieve the highest predictive power. The model included eight genes $(A P C, B M P 3$, BNC1, MESTV2, RASSF1A, TFPI2, and SFRP1-2) and patient's age $(>65)$. Although not quantitative, this model successfully distinguished malign from benign conditions with a sensitivity of $76 \%$ and a specificity of $83 \%$, independently of the tumor stage.

Finally, a recent study by Lehmann-Werman et al. revealed how cfDNA methylation patterns could be employed to detect tissue-specific cell death in individuals affected by PT or CP [21]. Array-based methylome data from cadaveric material of different organs showed that CUX2 and REG1A could be employed as pancreasspecific markers. While the former was preferentially unmethylated in ductal pancreatic cells, the latter was found unmethylated in both acinar and ductal pancreatic cells. cfDNA from matched plasma specimens showed high levels of CUX2 and REG1A in individuals with latestage PT or CP. Levels of these markers were lower as the grade of the lesion decreased, and it was minimum in healthy individuals, leading to the conclusion that tissue-specific cfDNA levels in the plasma directly reflect cell death, irrespective of the etiology of the disease. Besides, individuals affected by PT presented high levels of the ductal-specific marker $C U X 2$, whereas those affected by $\mathrm{CP}$ had higher levels of the acinar-ductal marker REG1A. In summary, hypomethylation of exocrine pancreas-specific cfDNA is detected in the blood of patients with PT and CP, mirroring the death of exocrine cells in these conditions.

\section{cfDNA methylation as a diagnostic marker of pre- neoplastic lesions}

Liquid biopsies are convenient and minimally invasive if compared to the more cumbersome and challenging procedure of tissue sampling. However, their utilization for the development of cfDNA methylation biomarkers presents some pitfalls. Despite the high tissue specificity of cfDNA methylation patterns [21] and the introduction of high-yield collection methods [65], the scarce abundance of disease-specific cfDNA remains a limiting factor. Thus, to achieve robust data analysis, the investigation has to be restricted to a few targets.

At present, what appears to be clear is that single epigenetic markers are seldom sufficient to distinguish pancreatic malign from benign or pre-neoplastic conditions. The simultaneous evaluation of more biomarkers has thus been investigated through systematic approaches. Today, prediction models are extensively used in a variety of biological fields [83, 84], and models integrating the DNA methylation status of target genes have been developed to provide prognostic estimates for individuals with PT [85]. Similarly, prediction models enabled the development of optimized aggregate biomarkers that combined different disease-specific informative targets, achieving high predictive power [82]. These models successfully distinguished PT from non-neoplastic conditions or CP with encouraging sensitivity and specificity. Besides, the utilization of stratification (e.g., age) or prognostic parameters may be a valid strategy to improve sensitivity. Recent evidence indicates that aberrant DNA methylation events correlate with longer patients' 


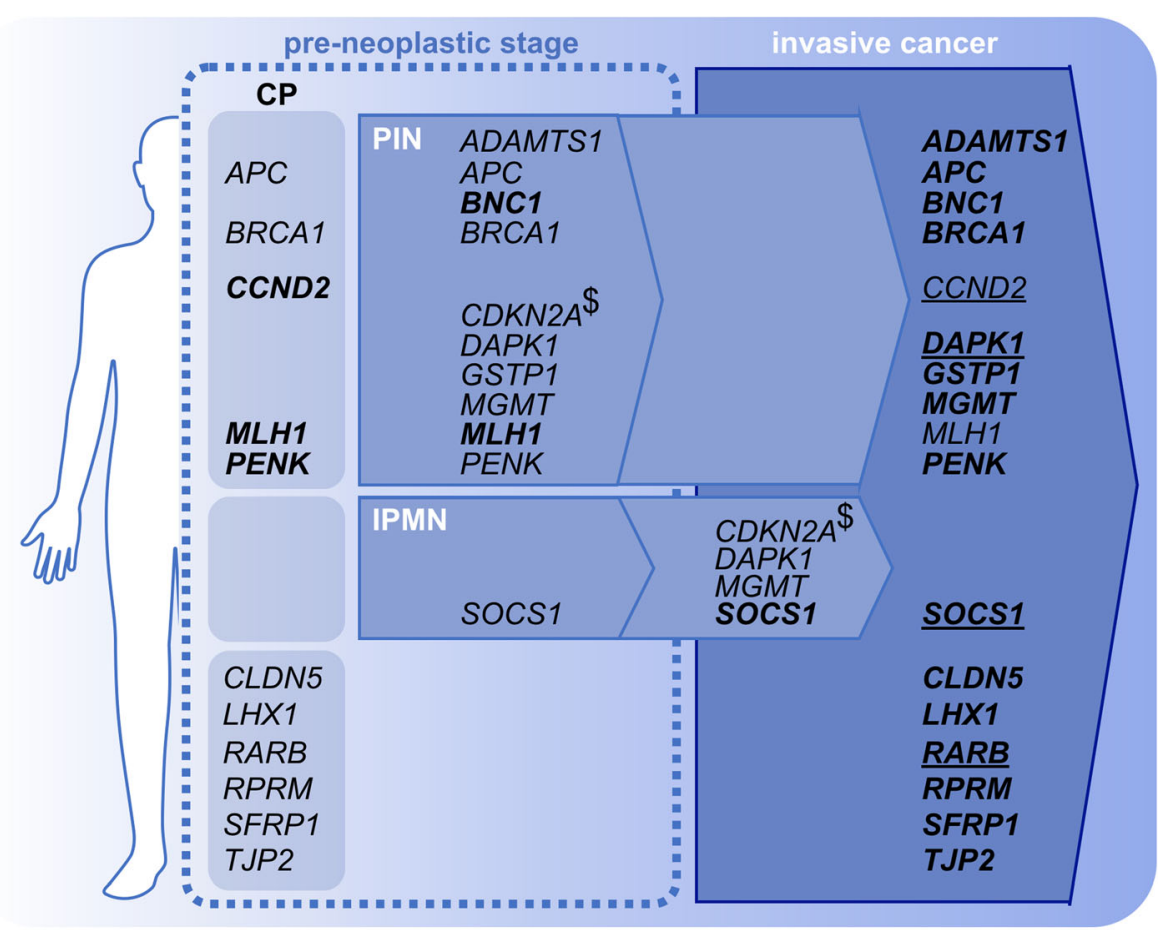

\$: p16INK4a specific region.

Fig. 2 Epigenetic landscape of pancreatic cancer. Graphical representation of the genes presenting different DNA methylation levels in CP, preneoplastic conditions, and invasive PT. Genes in bold present high DNA methylation levels, while normal formatting indicates that methylation is detected, though at lower levels. Underline genes present controversial results (e.g., hypermethylation is reported by different studies). \$:

p16INK4a specific region

survival [73]. As can be seen from Table 1, which shows the aggregate biomarkers discussed in this review, the grouped genes analyzed by Ligget's coworkers represent an excellent combination of sensitivity and specificity [80].

Figure 2 reports a schematic representation of the genes that may provide DNA methylation stage-specific information of pre-neoplastic conditions.

For some genes, DNA methylation may indeed mirror different stages of the disease progression (e.g., ADAMTS1 or SOCS1, in PINs or IPMNs, respectively) [55, 56, 65]. Other genes show dynamic DNA methylation levels that are increased by the persistent inflammatory environment (e.g., CP) [57], yet they are unmethylated at advanced stages of the disease (e.g., CCND2) [80]. On the other hand, genes such as APC, BRCA1, CLDN5, LHX1, RPRM, SFRP1, and TJP2, that progress to a state of increased methylation in invasive cancer, can be considered biomarkers of an aggressive stage of the disease [57, 63].

\section{Conclusions}

This review aimed to focus on the present advances in liquid biopsy for PT to establish predictive aggregate biomarkers that provide benefits to patients. Many candidate genes have been discussed in this review, the majority of which are hypermethylated as the pancreatic disease progresses. Interestingly, the use of new technologies and array-based screening employed to analyze DNA methylation status indicated that hypomethylation events, rather than hypermethylation, could be more informative because their occurrence appears to be independent of the inner tumor heterogeneity [78]. Besides, the decreasing cost of next-generation sequencing technologies offers an appealing option for methodological standardization. In conclusion, early diagnosis has the potential to allow prognosis prediction, tumor-stage monitoring, and provide personalized therapeutic strategies for patients suffering from a pancreatic disease.

\section{Additional file}

Additional file 1: Table S1. List of genes presenting differential DNA methylation between healthy pancreas, chronic pancreatitis or pancreatic cancer. CP: chronic pancreatitis; HiR: high PT-risk; IPMN: papillary mucinous neoplasms; PIN: pancreatic intraepithelial neoplasias; PT: pancreatic adenocarcinoma; PTX: pancreatic adenocarcinoma xenografts. Hyper:DNA hypermethylation; hypo: DNA hypomethylation; -: no change; inv.: invasive; *: non-significant; \#: cohort size <10; §: p16INK4a specific region; \$: p14ARF specific region; £: associated with longer survival. Grey 
background: genes analysed in both primary and liquid biopsies (XLS 57 $\mathrm{kb})$

\begin{abstract}
Abbreviations
5Aza-dC: 5-aza-2-deoxycytidine; AIP: Intraductal obstruction or autoimmune pancreatitis; CA19-9: Carbohydrate antigen 19-9; cfDNA: Cell-free DNA; CGI: CpG island; CP: Chronic pancreatitis; DMR: DNA-methylated region; DSB: DNA sequence Bisulfite; Hyper: Hypermethylation; Hypo: Hypomethylation; IPMN: Intraductal papillary mucinous neoplasm; MCA/RD: Methylation CpG island amplification/coupled with representational difference analysis; MCAM: Microarray platform; MeCap-seq: Methyl Capture sequencing; miR: MicroRNA; MSP: Methylation-specific PCR; MSREP: Methylation sensitive restriction enzyme-based qPCR; N: Normal; NGS: Next-generation sequencing; PIN: Pancreatic intraepithelial neoplasia; PNET: Pancreatic neuroendocrine tumor; PT: Pancreatic tumors; GMSP: Quantitative MSP; qPCR: Quantitative real-time PCR; RRBS: Reduced representation bisulfite sequencing; TSA: Trichostatin A; TSS: Transcription start site
\end{abstract}

\section{Gene names' abbreviations}

ADAMTS1: ADAM metallopeptidase with thrombospondin type 1 motif 1 ; ADCY5: adenylate cyclase 5: AGAP2: AGAP2 antisense RNA 1: ALX4: ALX homeobox 4; APC: APC regulator of WNT signaling pathway; ASCL2: Achaetescute family bHLH transcription factor 2; BMI1: BMI1 proto-oncogene, polycomb ring finger; $B M P 3$ : Bone morphogenetic protein 3; BNC1: Basonuclin 1; BNIP3: BCL2 interacting protein 3; BRCA1: BRCA1 DNA repair associated; BTBD6: BTB domain containing 6; C5orf38: Chromosome 5 open reading frame 38; CACNA1G: Calcium voltage-gated channel subunit alpha1 G; CACN A1H: Calcium voltage-gated channel subunit alpha1 H; CADM1: Cell adhesion molecule 1; CCND2: Cyclin D2; CCNG1: Cyclin G1; CD1D: CD1d molecule; CDH1: Cadherin 1; CDH3: Cadherin 3; CDKN1A: Cyclin-dependent kinase inhibitor 1A; CDKN1C: Cyclin-dependent kinase inhibitor 1C; CDKN2A: Cyclindependent kinase inhibitor 2A; CDKN2B: Cyclin-dependent kinase inhibitor 2B; CHFR: Checkpoint with forkhead and ring finger domains; CLDN4: Claudin 4; CLDN5: Claudin 5; CLEC11A: C-type lectin domain containing 11A; CNTN AP2: Contactin-associated protein-like 2; CTR9: CTR9 homolog, Paf1/RNA polymerase II complex component; CUX2: Cut-like homeobox 2; DAPK1: Death-associated protein kinase 1; DLX4: Distal-less homeobox 4; EFNA4: Ephrin A4; ELAVL2: ELAV like RNA binding protein 2; ELOVL4: ELOVL fatty acid elongase 4; EMX1: Empty spiracles homeobox 1; EP400: E1A binding protein p400; ESR1: Estrogen receptor 1; EVL: Enah/Nasp-like; EYA2: EYA transcriptional coactivator and phosphatase 2; FAM115A: Family with sequence similarity 115, member C (or TCAF1: TRPM8 channel associated factor 1); FOS: Fos proto-oncogene, AP-1 transcription factor subunit; FOXE1: Forkhead box E1; FOXF2/G1: Forkhead box G1 (or SNAR-G1: small NF90 (ILF3) associated RNA G1); FZD1: Frizzled class receptor 1; FZD2/ 7: Frizzled class receptor 2; GSTP1: Glutathione S-transferase pi 1; HIC1: HIC ZBTB transcriptional repressor 1; HIRIP3: HIRA-interacting protein 3; HPP1: Hyperpigmentation, progressive, 1; ID4: Inhibitor of DNA binding 4, HLH protein; IKZF1: IKAROS family zinc finger 1; IRF5: Interferon regulatory factor 5; IRX1: Iroquois homeobox 1; JUNB: JunB proto-oncogene, AP-1 transcription factor subunit; KCNK12: Potassium two pore domain channel subfamily K member 12; KDM6A: Lysine demethylase 6A; KMT5A: Lysine methyltransferase 5A; LCN2: Lipocalin 2; LHX1: LIM homeobox 1; MDFI: MyoD family inhibitor; MEST: Mesoderm-specific transcript; MGMT: 0-6methylguanine-DNA methyltransferase; MINT1/2/32/33: Methylated-in-tumor (MINT) loci; MIR10B: MicroRNA 10b; MIR124-1/-3: MicroRNA MIR124 family; MIR124-2HG: MIR124-2 host gene; MIR130B: MicroRNA 130b; MIR210: MicroRNA 210; MIR9-1: MicroRNA 9-1; MLH1: mutL homolog 1; MSLN: Mesothelin; MUC2: Mucin 2, oligomeric mucus/gel-forming; MYB: MYB proto-oncogene, transcription factor; MYOD1: Myogenic differentiation 1; NDN: Necdin, MAGE family member; NDRG4: NDRG family member 4; NEUROG1: Neurogenin 1; NEUROG3: Neurogenin 3; NPR3: Natriuretic peptide receptor 3; NPTX2: Neuronal pentraxin 2; PCDH17: Protocadherin 17; PDE4B: Phosphodiesterase 4B; PENK: Proenkephalin; PGK1: Phosphoglycerate kinase 1; PGR: Progesterone receptor; PITX2: Paired-like homeodomain 2; PLAU: Plasminogen activator, urokinase; PNMT: Phenylethanolamine Nmethyltransferase; PRKCB: Protein kinase $C$ beta; PRMT1: Protein arginine methyltransferase 1; PSCA: Prostate stem cell antigen; RARB: Retinoic acid receptor beta; RASSF1A: Ras association domain family member 1; RB1: RB transcriptional corepressor 1; REGTA: Regenerating family member 1 alpha; RPRM: Reprimo, TP53 dependent G2 arrest mediator homolog; S100A4: S100 calcium binding protein A4; SEPTIN9: Septin 9; SFN: Stratifin; SFRP1: Secreted frizzled-related protein 1; SFRP2: Secreted frizzled-related protein 2; SIM1: SIM bHLH transcription factor 1; SIM2: SIM bHLH transcription factor 2; SMARCA1: SWI/SNF related, matrix associated, actin dependent regulator of chromatin, subfamily a, member 1; SMOC2: SPARC-related modular calcium binding 2; SOCS1: Suppressor of cytokine signaling 1; SOX3: SRY-box transcription factor 3; SPARC: Secreted protein acidic and cysteine rich; SST: Somatostatin; ST14: Suppression of tumorigenicity 14; SYK: Spleenassociated tyrosine kinase; TAC1: Tachykinin precursor 1; TBX5: T-box transcription factor 5; TFAP2C: Transcription factor AP-2 gamma; TFF2: Trefoil factor 2; TFPI2: Tissue factor pathway inhibitor 2; THBS1: Thrombospondin 1; TIMP3: TIMP metallopeptidase inhibitor 3; TJP2: Tight junction protein 2; TP73: Tumor protein P73; TRADD: TNFRSF1A associated via death domain; TWIST1: Twist family bHLH transcription factor 1; UCHL1: Ubiquitin C-terminal hydrolase L1; VHL: von Hippel-Lindau tumor suppressor; VIM: Vimentin; VSTM2B: V-set and transmembrane domain containing 2B; WNT3: Wnt family member 3; WNT5A: Wnt family member 5A; WNT7A: Wnt family member 7A; ZBP: Z-DNA Binding Protein; ZBTB16: Zinc finger and BTB domain containing 16; ZNF415: Zinc finger protein 415

\section{Acknowledgements}

Not applicable.

\section{Author's contributions}

$\mathrm{FN}$, TA, and MV wrote the manuscript; FN and TA prepared the tables; FN and MV prepared the figures; TA and GF conceived the project. All authors reviewed the manuscript.

\section{Funding}

This work was funded by FFABR, University of Naples "Federico II" granted to TA (000020_FFABR_2017_ANGRISANO.TIZIANA).

Availability of data and materials

Not applicable.

Ethics approval and consent to participate

Not applicable.

\section{Consent for publication}

Not applicable.

\section{Competing interests}

The authors declare that they have no competing interests.

\section{Author details}

${ }^{1}$ Department of Biology, University of Naples Federico II, 80126 Naples, Italy. ${ }^{2}$ Biogem Scarl, Istituto di Ricerche Genetiche "Gaetano Salvatore", 83031 Ariano Irpino, Italy.

Received: 4 March 2019 Accepted: 16 August 2019 Published online: 06 September 2019

\section{References}

1. Majumder S, Chari ST. Chronic pancreatitis. The Lancet. 2016:387(10031): 1957-66. https://doi.org/10.1016/s0140-6736(16)00097-0.

2. Le Calvez-Kelm F, Foll M, Wozniak MB, Delhomme TM, Durand G, Chopard $P$, et al. Kras mutations in blood circulating cell-free DNA: a pancreatic cancer case-control. Oncotarget. 2016;7(48). https://doi.org/10.18632/ oncotarget.12386

3. Queneau PE, Adessi GL, Thibault P, Cleau D, Heyd B, Mantion G, et al. Early detection of pancreatic cancer in patients with chronic pancreatitis: diagnosticutility of a k-ras point mutation in the pancreatic juice. The American Journal of Gastroenterology. 2001;96(3):700-4. https://doi.org/1 0.1111/j.1572-0241.2001.03608.x.

4. Fukui T, Mitsuyama T, Takaoka M, Uchida K, Matsushita M, Okazaki K. Pancreatic cancer associated with autoimmune pancreatitis in remission. Internal Medicine. 2008:47(3):151-5. https://doi.org/10.2169/ internalmedicine.47.0334 
5. Yadav D, Lowenfels AB. The epidemiology of pancreatitis and pancreatic cancer. Gastroenterology. 2013;144(6):1252-61. https://doi.org/10.1053/j. gastro.2013.01.068.

6. Wolfgang $\mathrm{CL}$, Herman JM, Laheru DA, Klein AP, Erdek MA, Fishman EK, et al. Recent progress in pancreatic cancer. CA: A Cancer Journal for Clinicians 2013;63(5):318-48. https://doi.org/10.3322/caac.21190.

7. Malka D. Risk of pancreatic adenocarcinoma in chronic pancreatitis. Gut. 2002;51:849-52.

8. Ryan DP, Hong TS, Bardeesy N. Pancreatic adenocarcinoma. New England Journal of Medicine. 2014;371(11):1039-49. https://doi.org/10.1 056/nejmra1404198.

9. Hic M, llic I. Epidemiology of pancreatic cancer. World Journal of Gastroenterology. 2016;22(44):9694. https://doi.org/10.3748/wjg.v22.i44.9694

10. Jemal A, Siegel R, Ward E, Murray T, Xu J, Thun MJ. Cancer statistics, 2007. CA: A Cancer Journal for Clinicians. 2007;57(1):43-66. https://doi.org/1 0.3322/canjclin.57.1.43.

11. Raimondi S, Lowenfels AB, Morselli-Labate AM, Maisonneuve P, Pezzilli R. Pancreatic cancer in chronic pancreatitis; aetiology, incidence, and early detection. Best Practice Research Clinical Gastroenterology. 2010;24(3):349 -58. https://doi.org/10.1016/j.bpg.2010.02.007.

12. Yachida S, Jones S, Bozic I, Antal T, Leary R, Fu B, et al. Distant metastasis occurs late during the genetic evolution of pancreatic cancer. Nature. 2010; 467(7319):1114-7. https://doi.org/10.1038/nature09515.

13. Ni XG, Bai XF, Mao YL, Shao YF, Wu JX, Shan Y, et al. The clinical value of serum cea, ca19-9, and ca242 in the diagnosis and prognosis of pancreatic cancer. European Journal of Surgical Oncology (EJSO). 2005;31(2):164-9. https://doi.org/10.1016/j.ejso.2004.09.007.

14. Locker GY, Hamilton S, Harris J, Jessup JM, Kemeny N, Macdonald JS, et al. Asco 2006 update of recommendations for the use of tumor markers in gastrointestinal cancer. Journal of Clinical Oncology. 2006;24(33):5313-27. https://doi.org/10.1200/jco.2006.08.2644.

15. Goonetilleke KS, Siriwardena AK. Systematic review of carbohydrate antigen (ca 19-9) as a biochemical marker in the diagnosis of pancreatic cancer. European Journal of Surgical Oncology (EJSO). 2007;33(3):266-70. https:// doi.org/10.1016/j.ejso.2006.10.004.

16. Tada M. Detection or ras gene mutations in pancreatic juice and peripheral blood of patients with pancreatic adenocarcinoma. Cancer Research, ISSN. 1993). PubMed ID: 8495407:53:2472, 0008-4, 5472.

17. Adamo P, Cowley CM, Neal CP, Mistry V, Page K, Dennison AR, et al. Profiling tumour heterogeneity through circulating tumour DNA in patients with pancreatic cancer. Oncotarget. 2017;8(50). https://doi.org/10.18632/ oncotarget.20250.

18. Warton K, Samimi G. Methylation of cell-free circulating DNA in the diagnosis of cancer. Frontiers in Molecular Biosciences. 2015;2. https://doi. org/10.3389/fmolb.2015.00013.

19. Bettegowda C, Sausen M, Leary R, Kinde I, Wang Y, Agrawal N, et al. Detection of circulating tumor DNA in early- and late-stage human malignancies. Science Translational Medicine. 2014;6(224):224-2422424.

20. Schwarzenbach H, Hoon DSB, Pantel K. Cell-free nucleic acids as biomarkers in cancer patients. Nature Reviews Cancer. 2011;11(6):426-37. https://doi. org/10.1038/nrc3066.

21. Lehmann-Werman R, Neiman D, Zemmour H, Moss J, Magenheim J,

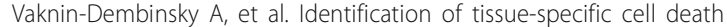
using methylation patterns of circulating DNA. PNAS; Proceedings of the National Academy of Sciences. 2016;113(13):1826-34.

22. Kisiel J, Raimondo M, Taylor W, Yab T, Mahoney D, Sun Z, et al. New DNA methylation markers for pancreatic cancer: Discovery, tissue validation, and pilot testing in pancreatic juice. Clin Cancer Res. 2015; 21(19):4473-81.

23. Imperiale TF, Ransohoff DF, Itzkowitz SH, Levin TR, Lavin P, Lidgard $G P$, et al. Multitarget stool DNA testing for colorectal-cancer screening. New England Journal of Medicine. 2014;370(14):1287-97. https://doi.org/10.1056/nejmoa1311194.

24. Lidgard GP, Domanico MJ, Bruinsma JJ, Light J, Gagrat ZD, OldhamHaltom RL, et al. Clinical performance of an automated stool DNA assay for detection of colorectal neoplasia. Clinical Gastroenterology and Hepatology. 2013;11(10):1313-8. https://doi.org/10.1016/j.cgh.2 013.04.023.

25. Ying H, Dey P, Yao W, Kimmelman AC, Draetta GF, Maitra A, DePinho RA. Genetics and biology of pancreatic ductal adenocarcinoma. Genes Development. 2016;30(4):355-85. https://doi.org/10.1101/gad.275776.115.
26. Aguirre AJ. Activated Kras and Ink4a/Arf deficiency cooperate to produce metastatic pancreatic ductal adenocarcinoma. Genes Development. 2003; 17(24):3112-26. https://doi.org/10.1101/gad.1158703.

27. Muniz V, Barnes JM, Paliwal S, Zhang X, Tang X, Chen S, et al. The ARF tumor suppressor inhibits tumor cell colonization independent of p53 in a novel mouse model of pancreatic ductal adenocarcinoma metastasis. Molecular Cancer Research. 2011;9(7):867-77.

28. Wei J, Zaika E, Zaika A. p53 Family: role of protein isoforms in human cancer. Journal of Nucleic Acids. 2012;687359. https://doi.org/1 $0.1155 / 2012 / 687359$

29. Troiano A, Lomoriello IS, di Martino O, Fusco S, Pollice A, Vivo M, et al. Y -box binding protein-1 is part of a complex molecular network linking np63 to the pi3k/akt pathway in cutaneous squamous cell carcinoma. Journal of Cellular Physiology. 2015;230(9):2067-74.

30. di Martino O, Troiano A, Guarino AM, Pollice A, Vivo M, La Mantia G, et al. np63 controls yb-1 protein stability: evidence on yb-1 as a new player in keratinocyte differentiation. Genes to Cells. 2016.

31. Vivo M, Fontana R, Ranieri M, Capasso G, Angrisano T, Pollice A, et al. p14arf interacts with the focal adhesion kinase and protects cells from anoikis. Oncogene. 2017;36(34):4913-28. https://doi.org/10.1038/onc.2017.104.

32. Delpu Y, Hanoun N, Lulka H, Sicard F, Selves J, Buscail L, et al. Genetic and epigenetic alterations in pancreatic carcinogenesis. Current Genomics. 2011; 12(1):15-24. https://doi.org/10.2174/138920211794520132.

33. Rooney SL, Shi J. Intraductal tubulopapillary neoplasm of the pancreas: an update from a pathologist's perspective. Archives of Pathology Laboratory Medicine. 2016;140(10):1068-73. https://doi.org/10.5858/arpa.2016-0207-ra.

34. Lewis MA, Yao JC. Molecular pathology and genetics of gastrointestinal neuroendocrine tumours. Current Opinion in Endocrinology Diabetes and Obesity. 2014;21(1):22-7. https://doi.org/10.1097/med.0000000000000033.

35. Burns WR, Edil BH. Neuroendocrine pancreatic tumors: Guidelines for management and update. Current Treatment Options in Oncology. 2012; 13(1):24-34. https://doi.org/10.1007/s11864-011-0172-2.

36. Schenkel LC, Kernohan KD, McBride A, Reina D, Hodge A, Ainsworth PJ, et al. Identification of epigenetic signature associated with alpha thalassemia/ mental retardation x-linked syndrome. Epigenetics Chromatin. 2017;10(1). https://doi.org/10.1186/s13072-017-0118-4.

37. Cai J, Chen J, Zhang W, Yang P, Zhang C, Li M, et al. Loss of atrx, associated with DNA methylation pattern of chromosome end, impacted biological behaviors of astrocytic tumors. Oncotarget. 2015;6(20). https://doi.org/10.1 8632/oncotarget.3906.

38. TCGA-Network. Comprehensive genomic characterization of head and neck squamous cell carcinomas. Nature. 2015;517(7536):576-82. https://doi.org/1 0.1038 /nature14129.

39. Raghuraman S, Donkin I, Versteyhe S, Barrès R, Simar D. The emerging role of epigenetics in inflammation and immunometabolism. Trends in Endocrinology and Metababolism. 2016;27(11):782-95. https://doi.org/10.1 016/j.tem.2016.06.008.

40. Maiuri AR, O'Hagan HM. Interplay between inflammation and epigenetic changes in cancer. Progress in Molecular Biology and Translational Science. 2016;144:69-117. https://doi.org/10.1016/bs.pmbts.2016.09.002.

41. Rasmussen KD, Helin K. Role of tet enzymes in DNA methylation, development, and cancer. Genes Development. 2016;30(7):733-50. https:// doi.org/10.1101/gad.276568.115.

42. Angrisano T, Pero R, Brancaccio M, Coretti L, Florio E, Pezone A, et al. Cyclical DNA methylation and histone changes are induced by lps to activate cox-2 in human intestinal epithelial cells. PLOS ONE. 2016;11(6): 0156671. https://doi.org/10.1371/journal.pone.0156671.

43. Angrisano T, Pero R, Paoletti I, Keller S, Lembo L, Baroni A, et al. Epigenetic regulation of il-8 and -defensin genes in human keratinocytes in response to Malassezia furfur. Journal of Investigative Dermatology. 2013;133(8):2101 -4. https://doi.org/10.1038/jid.2013.143.

44. Chiariotti L, Angrisano T, Keller S, Florio E, Affinito O, Pallante P, et al. Epigenetic modifications induced by helicobacter pylori infection through a direct microbe-gastric epithelial cells cross-talk. Medical Microbiology and Immunology. 2013;202(5):327-37. https://doi.org/10.1007/s00430-013-0301-6.

45. Gerdes B, Ramaswamy A, Kersting M, Ernst M, Lang S, Schuermann M, et al. p16ink4aalterations in chronic pancreatitis-indicator for high-risk lesions for pancreatic cancer. Surgery. 2001;129(4):490-7. https://doi.org/10.1016/ s0039-6060(01)01158-8.

46. Guerra C, Collado M, Navas C, Schuhmacher AJ, Hernandez-Porras I, Canamero $\mathrm{M}$, et al. Pancreatitis-induced inflammation contributes to 
pancreatic cancer by inhibiting oncogene-induced senescence. Cancer Cell. 2011;19(6):728-39. https://doi.org/10.1016/j.ccr.2011.05.011.

47. Kinugawa Y, Uehara T, Sano K, Matsuda K, Maruyama Y, Kobayashi Y, et al. Methylation of tumor suppressor genes in autoimmune pancreatitis. Pancreas. 2017;46(5):614-8. https://doi.org/10.1097/mpa.0000000000000804.

48. Pharo HD, Andresen K, Berg KCG, Lothe RA, Jeanmougin M, Lind GE. A robust internal control for high-precision DNA methylation analyses by droplet digital PCR. Clinical Epigenetics. 2018;10(24). https://doi.org/10.1186/ s13148-018-0456-5.

49. Baylin SB, Jones PA. A decade of exploring the cancer epigenome-biological and translational implications. Nature Reviews Cancer. 2011;11(10):726-34. https://doi.org/10.1038/nrc3130.

50. Ueki T, Toyota M, Sohn T, Yeo C, Issa JP, Hruban R, et al. Hypermethylation of multiple genes in pancreatic adenocarcinoma. Cancer Research. 2000; 60(7):1835-9 PUBMED.

51. Ueki T, Totyota M, Skinner H, Walter KM, Yeo CJ, Issa JP, et al. Identification and characterization of differentially methylated $\mathrm{CpG}$ islands in pancreatic carcinoma. Cancer Research. 2001;61(23) [PUBMED].

52. Ueki T, Walter KM, Skinner H, Jaffee E, Hruban RH, Goggins M. Aberrant CpG island methylation in cancer cell lines arises in the primary cancers from which they were derived. Oncogene. 2002;21(13):2114-7. https://doi.org/1 0.1038/sj.onc.1205275

53. Fukushima $N$, Sato $N$, Ueki T, Rosty C, Walter KM, Wilentz RE, et al. Aberrant methylation of preproenkephalin and p16 genes in pancreatic intraepithelial neoplasia and pancreatic ductal adenocarcinoma. The American Journal of Pathology. 2002;160(5):1573-81. https://doi.org/10.1 016/s0002-9440(10)61104-2.

54. Li G, Ji Y, Liu C, Li J, Zhou Y. Reduced levels of p15ink4b, p16ink4a, p21cip1 and p27kip1 in pancreatic carcinoma. MolecularMedicine Reports. 2012;5(4): 1106-10. https://doi.org/10.3892/mmr.2012.771.

55. Fukushima N, Sato N, Sahin F, Su GH, Hruban RH, Goggins M. Aberrant methylation of suppressor of cytokine signalling-1 (socs-1) gene in pancreatic ductal neoplasms. British Journal of Cancer. 2003;89(2):338-43. https://doi.org/10.1038/sj.bjc.6601039.

56. House MG, Guo M, lacobuzio-Donahue C, Herman JG. Molecular progression of promoter methylation in intraductal papillary mucinous neoplasms (IPMN) of the pancreas. Carcinogenesis. 2003;24(2):193-8. https:// doi.org/10.1093/carcin/24.2.193.

57. Peng DF, Sawada M, Hiraoka N, Ushijima S, Hirohashi S, et al. DNA methylation of multiple tumor-related genes in association with overexpression of DNA methyltransferase 1 (DNMT1) during multistage carcinogenesis of the pancreas. Carcinogenesis. 2006;27(6):1160-8. https:// doi.org/10.1093/carcin/bgi361.

58. Zhao Y, Sun J, Zhang H, Guo S, Gu J, Wang W, et al. High-frequency aberrantly methylated targets in pancreatic adenocarcinoma identified via global DNA methylation analysis using methylcap-seq. Clinical Epigenetics. 2014;6(1):18. https://doi.org/10.1186/1868-7083-6-18.

59. Sato N, Maitra A, Fukushima N, van Heek NT, Matsubayashi H, et al. Frequent hypomethylation of multiple genes overexpressed in pancreatic ductal adenocarcinoma. Cancer Res. 2003;63(14):4158-66 [PUBMED].

60. Jones S, Zhang X, Parsons DW, Lin JCH, Leary RJ, Angenendt P, et al. Core signaling pathways in human pancreatic cancers revealed by global genomic analyses. Science. 2008;321(5897):1801-6. https://doi.org/10.1126/ science.1164368

61. Vincent A, Omura N, Hong SM, Jaffe A, Eshleman J, Goggins M. Genome -wide analysis of promoter methylation associated with gene expression profile in pancreatic adenocarcinoma. Clin Cancer Res. Clinical Cancer Research. 2011;17(13):4341-54

62. Tan AC, Jimeno A, Lin SH, Wheelhouse J, Chan F, Solomon A, et al. Characterizing DNA methylation patterns in pancreatic cancer genome. Molecular Oncology. 2009;3(5-6):425-38. https://doi.org/10.1016/j.molonc.2009.03.004.

63. Sato N, Fukushima N, Maitra A, Matsubayashi H, Yeo C, Cameron J, et al. Discovery of novel targets for aberrant methylation in pancreatic carcinoma using high-throughput microarrays. Cancer Research. 2003; 63(13):3735-42 PUBMED.

64. Omura N, Li C-P, Li A, Hong S-M, Walter K, Jimeno A, Hidalgo M, Goggins M. Genome-wide profiling of methylated promoters in pancreatic adenocarcinoma. Cancer biology \& therapy. 2008;7(7):1146-56.

65. Yi J, Guzzetta A, Bailey V, Downing S, VanNeste L, Chiappinelli K, et al. Novel methylation biomarker panel for the early detection of pancreatic cancer. Clinical Cancer Research. 2013;19(23):6544-55.
66. Wang P, Chen L, Zhang J, Chen H, Fan J, Wang K, et al. Methylationmediated silencing of the mir-124 genes facilitates pancreatic cancer progression and metastasis by targeting rac1. Oncogene. 2013;33(4):514-24. https://doi.org/10.1038/onc.2012.598.

67. Chan AY, Coniglio SJ, Chuang Y-y. Michaelson D, KnausUG, Philips MR, et al. Roles of the rac1 and rac3 GTPases in human tumor cell invasion. Oncogene. 2005;24(53):7821-9. https://doi.org/10.1038/sj.onc.1208909.

68. Heid I, Lubeseder-Martellato C, Sipos B, Mazur PK, Lesina M, Schmid RM, et al. Early requirement of rac1 in a mouse model of pancreatic cancer. Gastroenterology. 2011;141(2):719-7307. https://doi.org/10.1053/j. gastro.2011.04.043.

69. Nakata K, Ohuchida K, Mizumoto K, Kayashima T, Ikenaga N, Sakai H, et al. Microrna-10b is overexpressed in pancreatic cancer, promotes its invasiveness, and correlates with a poor prognosis. Surgery. 2011;150(5): 916-22. https://doi.org/10.1016/j.surg.2011.06.017.

70. Ma L, Reinhardt F, Pan E, Soutschek J, Bhat B, Marcusson EG, et al. Therapeutic silencing of mir-10b inhibits metastasis in a mouse mammary tumor model. Nature Biotechnology. 2010;28(4):341-7. https://doi.org/10.1038/nbt.1618.

71. Takikawa T, Masamune A, Hamada S, Nakano E, Yoshida N, Shimosegawa T. mir-210 regulates the interaction between pancreatic cancer cells and stellate cells. Biochemical and Biophysical Research Communications. 2013; 437(3):433-9. https://doi.org/10.1016/j.bbrc.2013.06.097.

72. Devlin C, Greco S, Martelli F, Ivan M. mir-210: More than a silent player in hypoxia. IUBMB Life. 2011. https://doi.org/10.1002/iub.427.

73. Thompson MJ, Rubbi L, Dawson DW, Donahue TR, Pellegrini M. Pancreatic cancer patient survival correlates with DNA methylation of pancreas development genes. PLOS ONE. 2015;10(6):0128814. https://doi.org/10.1371/ journal.pone.0128814.

74. Zhao G, Zhang J-g, Shi Y, Qin Q, Liu Y, Wang B, et al. Mir-130b is a prognostic marker and inhibits cell proliferation and invasion in pancreatic cancer through targeting stat3. PLoS ONE. 2013;8(9):73803. https://doi.org/1 0.1371/journal.pone.0073803.

75. Colangelo T, Fucci A, Votino C, Sabatino L, Pancione M, Laudanna C, et al. Microrna-130b promotes tumor development and is associated with poor prognosis in colorectal cancer. Neoplasia. 2013;15(9):1086-99. https://doi. org/10.1593/neo.13998.

76. Matsubayashi H, Canto M, Sato N, Klein A, Abe T, Yamashita K, et al. DNA methylation alterations in the pancreatic juice of patients with suspected pancreatic disease. Cancer Res. 2006;66(2):1208-17.

77. Melnikov AA, Scholtens D, Talamonti MS, Bentrem DJ, Levenson W. Methylation profile of circulating plasma DNA in patients with pancreatic cancer. Journal of Surgical Oncology. 2009;99(2):119-22. https://doi.org/10.1002/jso.21208.

78. Melnikov AA, Scholtens DM, Wiley EL, Khan SA, Levenson W. Array -based multiplex analysis of DNA methylation in breast cancer tissues. The Journal of Molecular Diagnostics. 2008;10(1):93-101. https://doi. org/10.2353/jmoldx.2008.070077.

79. Matsubayashi H, Sato N, Fukushima N, Yeo CJ, Walter KM, Brune K, Sahin F, Hruban $\mathrm{RH}$, Goggins M. Methylation of cyclin D2 is observed frequently in pancreatic cancer but is also an age-related phenomenon in gastrointestinal tissues. Clinical Cancer Research. 2003;9(4):1446-52. PUBMED.

80. Liggett T, Melnikov A, Yi Q, Replogle C, Brand R, Kaul K, et al. Differential methylation of cell-free circulating DNA among patients with pancreatic cancer versus chronic pancreatitis. Cancer. 2010;116(7):1674-80. https://doi. org/10.1002/cncr.24893.

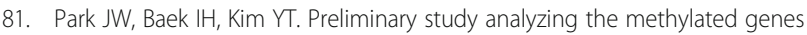
in the plasma of patients with pancreatic cancer. Scandinavian Journal of Surgery. 2012;101(1):38-44. https://doi.org/10.1177/145749691210100108.

82. Henriksen SD, Madsen PH, Larsen AC, Johansen MB, Drewes AM, Pedersen IS, et al. Cell-free DNA promoter hypermethylation in plasma as a diagnostic marker for pancreatic adenocarcinoma. Clinical Epigenetics. 2016;8(1). https://doi.org/10.1186/s13148-016-0286-2.

83. Bleicher M, Burigo L, Durante M, Herrlitz M, Kraemer M, Mishustin I, et al. Nanolesions induced by heavy ions in human tissues: experimental and theoretical studies. Beilstein Journal of Nanotechnology. 2012;3:556-63. https://doi.org/10.3762/bjnano.3.64.

84. Loeb D, Lengert N, Chagin VO, Reinhart M, Casas-Delucchi CS, Cardoso MC, et al. $3 \mathrm{~d}$ replicon distributions arise from stochastic initiation and domino -like DNA replication progression. Nature Communications. 2016;7(1). https://doi.org/10.1038/ncomms11207. 
85. Dam Henriksen S, Henning Madsen P, Christian Larsen A, Berg Johansen M, Søkilde Pedersen I, Krarup H, et al. Cell-free DNA promoter

hypermethylation in plasma as a predictive marker for survival of patients with pancreatic adenocarcinoma. Oncotarget. 2017. https://doi.org/10.1 8632/oncotarget.21397.

\section{Publisher's Note}

Springer Nature remains neutral with regard to jurisdictional claims in published maps and institutional affiliations.

Ready to submit your research? Choose BMC and benefit from:

- fast, convenient online submission

- thorough peer review by experienced researchers in your field

- rapid publication on acceptance

- support for research data, including large and complex data types

- gold Open Access which fosters wider collaboration and increased citations

- maximum visibility for your research: over $100 \mathrm{M}$ website views per year

At $\mathrm{BMC}$, research is always in progress.

Learn more biomedcentral.com/submissions 